\title{
Lordoplasty: midterm outcome of an alternative augmentation technique for vertebral fractures
}

\author{
Sven Hoppe, MD, ${ }^{1}$ Mathias Budmiger, ${ }^{1}$ Philipp Bissig, MD, ${ }^{1}$ Emin Aghayev, MD, ${ }^{2}$ and \\ Lorin M. Benneker, MD'
} 1Department of Orthopedic Surgery, Inselspital, and ${ }^{2}$ Institute for Evaluative Research in Medicine, University of Bern,
Switzerland

\begin{abstract}
OBJECTIVE Vertebroplasty and balloon kyphoplasty are effective treatment options for osteoporotic vertebral compression fractures but are limited in correction of kyphotic deformity. Lordoplasty has been reported as an alternative, cost-effective, minimally invasive, percutaneous cement augmentation technique with good restoration of vertebral body height and alignment. The authors report on its clinical and radiological midterm results.

METHODS A retrospective review was conducted of patients treated with lordoplasty from 2002 to 2014 . Inclusion criteria were clinical and radiological follow-up evaluations longer than 24 months. Radiographs were accessed regarding initial correction and progressive loss of reduction. Complications and reoperations were recorded. Actual pain level, pain relief immediately after surgery, autonomy, and subjective impression of improvement of posture were assessed by questionnaire.
\end{abstract}

RESULTS Sixty-five patients (46 women, 19 men, age range 38.9-86.2 years old) were treated with lordoplasty for 69 vertebral compression and insufficiency fractures. A significant correction of the vertebral kyphotic angle (mean $\left.13^{\circ}\right)$ and segmental kyphotic angle (mean $11^{\circ}$ ) over a mean follow-up of 33 months (range 24-108 months) was achieved ( $p<$ 0.001). On average, pain was relieved to $90 \%$ of the initial pain level. In $24 \%$ of the 65 patients a second spinal intervention was necessary: 16 distant (24.6\%) and 7 adjacent (10.8\%) new osteoporotic fractures, 4 instrumented stabilizations (6.2\%), 1 new adjacent traumatic fracture (1.5\%), and 1 distant microsurgical decompression (1.5\%). Cement leakage occurred in $10.4 \%$ but was only symptomatic in 1 case.

CONCLUSIONS Lordoplasty appeared safe and effective in midterm pain alleviation and restoration of kyphotic deformity in osteoporotic compression and insufficiency fractures. The outcomes of lordoplasty are consistent with other augmentation techniques.

http://thejns.org/doi/abs/10.3171/2015.10.SPINE151016

KEY WORDS lordoplasty; vertebral compression fracture; osteoporosis; cement augmentation; kyphotic correction; technique

I $\mathrm{N}$ the past 2 decades, the interventional treatment of vertebral compression fractures (VCFs) has undergone enormous progress. Different approaches were developed to restore vertebral body height, correct kyphotic posture, stabilize the fractured vertebra, and reduce pain. Vertebroplasty, balloon kyphoplasty (BKP), and vertebral body stenting (VBS) are probably the most frequently reported and used approaches. ${ }^{7,12}$ Despite successful pain relief in $80 \%-90 \% 1,10,26$ and improvement of quality of life with these approaches, ${ }^{9,27}$ they all have certain limitations. Vertebroplasty is the least expensive procedure but involves a limited restoration of vertebral body height and correction of the kyphotic deformity. ${ }^{13,24}$ BKP and VBS were specifically conceived for restoration of vertebral body height by inflation of 1-2 balloons inside the fractured vertebra prior to cement injection. In contrast to BKP, VBS applies expandable stents in addition to balloons to prevent height loss after the deflation of the balloons. ${ }^{9,18,30}$ Although both latter techniques are effective in terms of pain relief and partial correction of the kyphotic deformity, their high product cost limits their distribution. ${ }^{9,14,15}$

In 2006, Orler et al. introduced an alternative surgical approach called lordoplasty. ${ }^{25}$ The lordoplasty concept

ABBREVIATIONS BKA = bisegmental kyphotic angle; BKP = balloon kyphoplasty; SKA = segmental kyphotic angle; VBS = vertebral body stenting; $\mathrm{VCF}=$ vertebral compression fracture; VKA = vertebral kyphotic angle.

SUBMITTED August 24, 2015. ACCEPTED October 30, 2015.

INCLUDE WHEN CITING Published online February 19, 2016; DOI: 10.3171/2015.10.SPINE151016. 
allowed good correction of kyphosis and restoration of vertebral body height at a reasonably low cost. The lordoplasty concept is based on the principles of ligamentotaxis, similar to those in open reduction of traumatic fractures. During lordoplasty, the fractured and adjacent vertebrae are instrumented bipedicularly with cannulas, and the fracture is reduced indirectly by applying a lordotic moment over the cannulas to the adjacent vertebrae prior to cement augmentation of the fractured level (Fig. 1). The technique may be combined with BKP or VBS to maximize the potential for restoration of vertebral body height and, for example, to allow a minimally invasive treatment of incomplete burst fractures (AO/Magerl Type A3.1). ${ }^{6}$ Several years after the initial report, there are very few clinical studies on lordoplasty that have been published. ${ }^{14,15}$ In our department, the lordoplasty approach has been used for 10 years. The aim of this study was to analyze the midterm results of our case series.

\section{Methods}

The study design was a retrospective analysis of prospectively collected data on a case series of a Level 1 trauma center (Inselspital, University of Bern).

\section{Patient Sample}

Since January 2002, 301 patients have been treated with lordoplasty at our department. The indications for surgery were acute (pain < 6 weeks) atraumatic osteoporotic VCF, and traumatic insufficiency fractures (fall from standing height) of A1.2, A1.3, or A3.1 type, according to the classification system of $\mathrm{AO} / \mathrm{Magerl}$ et al. ${ }^{23}$ with a vertebral kyphotic angle (VKA) on standing radiographs of more than $15^{\circ}$.

The inclusion criteria for the analysis were a minimum follow-up time of 24 months, a complete radiological documentation consisting of standing anteroposterior and lateral radiographs preoperatively and postoperatively, as well as at the latest follow-up evaluation. Exclusion criteria were traumatic, not osteoporotic/osteopenia-associated fractures, incomplete radiological analysis at some stage, and intraoperative use of balloon-assisted procedures (kyphoplasty/stentoplasty) for reduction of the fracture.

\section{Surgical Technique}

The surgery was performed while the patients were under general anesthesia. In cases in which general anesthesia is contraindicated, the surgery can be performed with local anesthesia as well, similar to vertebroplasty. All procedures were performed using intraoperative fluoroscopy. The fractured and the adjacent vertebrae are bipedicular instrumented with vertebroplasty cannulas. After vertebroplasty of the adjacent vertebrae (above and below the fractured vertebra), the fracture is reduced indirectly by applying a lordotic moment via the cannulas in these adjacent vertebrae, using the facets as a hypomochlion/ fulcrum (Fig. 1). After maximal possible reduction, the fractured vertebra can be augmented, aiming for an endplate-to-endplate filling pattern. In all cases, standard polymethylmethacrylate cement (Vertecem, Synthes AG) was used according to the product specifications. In none of the presented cases was a balloon-assisted procedure (kyphoplasty or stentoplasty) additionally added for reduction purposes.

\section{Radiological Assessment}

The VKA, segmental kyphotic angle (SKA), and bisegmental kyphotic angle (BKA) were measured at baseline, immediately postoperatively, and at the latest follow-up evaluation. ${ }^{19}$ VKA is defined as the angle between the superior and inferior endplate of the fractured vertebra, SKA as the angle between the inferior endplate of the fractured vertebra and the superior endplate of the cranial adjacent vertebra, and BKA as the angle between the superior endplate of the cranial adjacent vertebra and the inferior endplate of the caudal adjacent vertebra. The occurrence of cement leakage and incidence of new VCF were evaluated based on postoperative radiographs. Three independent observers measured all angles and a consensus method was used in differences greater than $3^{\circ}$. All measurements were performed using digital DICOM images (PACS Sectra Workstation IDS7, Sectra).

\section{Clinical Assessment}

Surgical duration and frequencies of complications and reoperations were captured. For subjective evaluation, the
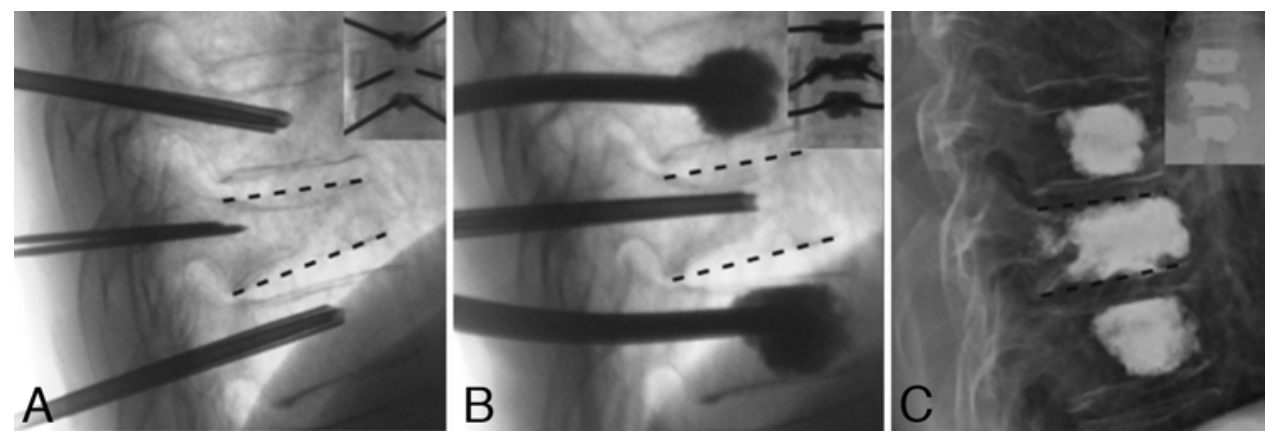

FIG. 1. Images obtained in a 76-year-old woman with an osteoporotic T-10 compression fracture. A: Intraoperative fluoroscopy shows a VKA of $13^{\circ}$ persists in the prone position. B: Intraoperative fluoroscopy showing application of a lordotic moment via the cannulas placed in the adjacent vertebrae leading to a VKA of $2^{\circ}$. In this osteoporotic fracture the adjacent levels were cemented prior to the repositioning. C: Standing radiograph obtained 1 year after the intervention showing no loss of reduction. Dashed lines represent the endplates of the fractured vertebra. 
following were extracted: 1) amount of perceived pain relief after surgery; 2) actual pain intensity on the visual analog scale at the time of last follow-up; 3) self-dependence; 4) improvement of posture after surgery; and 5) whether the patient would undergo the lordoplasty again.

\section{Statistical Analyses}

Continuous variables are shown using means and standard deviations, and categorized variables using proportions. The Wilcoxon signed-rank test was used for the comparison of outcome changes over time. All statistical analyses were conducted using SAS (version 9.4, SAS Institute Inc.) with an $\alpha$ level of 0.05 .

\section{Results}

The mean follow-up duration was 33 months (range 24-108 months). Sixty-five patients (46 women, 19 men, age range 38.9-86.2 years old) met inclusion criteria and were included in the analysis. In $4(6 \%)$ of the 65 patients, lordoplasty was performed at 2 adjacent levels; thus the total number of treated vertebrae was 69 (Table 1). Thirtyfour fractures were spontaneous atraumatic osteoporotic fractures, and 35 were traumatic "insufficiency" factures (fall from standing height).

The mean surgical duration was 55 minutes (range 3090 minutes). No relevant blood loss was observed. None of the patients experienced an intraoperative complication, such as cement leakage into the spinal canal or pulmonary cement embolism.

The VKA significantly decreased from $22^{\circ}$ at baseline to $9^{\circ}$ immediately postoperatively $(\mathrm{p}<0.001)$. A marginal loss of reduction $\left(0.3^{\circ}\right)$ of VKA was noted at the latest follow-up evaluation (Table 2). Similarly, SKA and BKA also improved immediately postoperatively from $21^{\circ}$ and $23^{\circ}$ to $10^{\circ}$ and $15^{\circ}$, respectively, and remained clinically stable with $11^{\circ}$ and $17^{\circ}$, respectively, measured at followup (Table 2).

A cement leakage into segmental veins or paraspinous tissue was noted in 7 vertebrae (10\%). One of these patients, a 73-year-old woman with a type A 3.1 fracture of $\mathrm{T}-12$, showed a cement leakage through the fracture gap that was symptomatic, causing an irritation of the twelfth intercostal nerve. The patient was treated with a costovertebral joint injection with local anesthetics and steroids, which led to an immediate and lasting pain relief.

In $23(35 \%)$ of the 65 patients, 28 secondary interventions on the spine were required after the lordoplasty during the cumulative observation period of nearly 9 years. The indications for new surgery (based on the number of patients, not fractures) were as follows: a new distant osteoporotic fracture $(\mathrm{n}=16,24.6 \%)$, a new adjacent osteoporotic fracture ( $\mathrm{n}=7,11 \%$; Fig. 2$)$, an instrumented fusion due to instability $(n=4,6 \%)$, and a new adjacent traumatic fracture $(n=1,2 \%)$.

Of the 65 patients, the data of 52 patients could be assessed for subjective evaluation, whereas in 13 the data were incomplete. The reasons for incomplete data were severe dementia $(n=1)$, loss of the questionnaire $(n=6)$, and noncompliance of the medical staff $(n=6)$. Of 52 patients, 37 (71\%) reported retrospectively that they per-
TABLE 1. Fracture levels of 69 treated vertebrae

\begin{tabular}{cc}
\hline Disc Level & No. of Vertebrae \\
\hline T-5 & 3 \\
\hline T-6 & 1 \\
\hline T-7 & 3 \\
\hline T-8 & 5 \\
\hline T-9 & 3 \\
\hline T-10 & 3 \\
\hline T-11 & 6 \\
\hline T-12 & 26 \\
\hline L-1 & 16 \\
\hline L-3 & 2 \\
\hline L-4 & 1 \\
\hline Total & 69 \\
\hline
\end{tabular}

ceived a $100 \%$ reduction of pain due to the intervention, 5 patients $(10 \%)$ perceived a $90 \%$ reduction, 9 patients $(17 \%)$ between $50 \%$ and $80 \%$ reduction, and 1 patient $(2 \%)$ no reduction of pain. The mean current level of back pain was 2.1 points (range 1-7 points) on the visual analog scale. Ninety-six percent of patients $(n=50)$ were self-dependent. Two patients (4\%) remained dependent after surgery and required further conservative therapy due to chronic, immobilizing back pain. Eighty-eight percent of patients $(n=46)$ were convinced that lordoplasty improved their posture substantially, and $98 \%$ of the patients $(n=51)$ would undergo this intervention again.

\section{Discussion}

The study focused on the midterm outcome of osteoporotic vertebral compression and simple traumatic fractures treated by lordoplasty. In addition to pain relief and prevention of a segmental collapse, restoration of the sagittal alignment is one of the main intentions of the technique.

The analysis showed a mean immediate postoperative reduction of the VKA and SKA of $13^{\circ}$ and $10^{\circ}$, respectively, which remained stable over 33 months with a marginal

TABLE 2. Changes in angles between time points in 69 fractures

\begin{tabular}{llcc}
\hline \multicolumn{1}{c}{ Angle } & Mean $\left(^{\circ}\right)$ & SD $\left(^{\circ}\right)$ & p Value \\
\hline VKA & & & \\
\hline Baseline to postop & 13 & 7.1 & $<0.001$ \\
\hline Baseline to follow-up & 12.7 & 6.6 & $<0.001$ \\
\hline Postop to follow-up & 0.3 & 4.1 & 0.046 \\
\hline SKA & & & \\
\hline Baseline to postop & 11 & 7.7 & $<0.001$ \\
\hline Baseline to follow-up & 10.4 & 8.1 & $<0.001$ \\
\hline Postop to follow-up & -0.7 & 4.6 & 0.09 \\
\hline BKA & & & \\
\hline Baseline to postop & 7.4 & 7.2 & $<0.001$ \\
\hline Baseline to follow-up & 5.2 & 8.7 & $<0.001$ \\
\hline Postop to follow-up & 2.1 & 7.2 & 0.016 \\
\hline
\end{tabular}




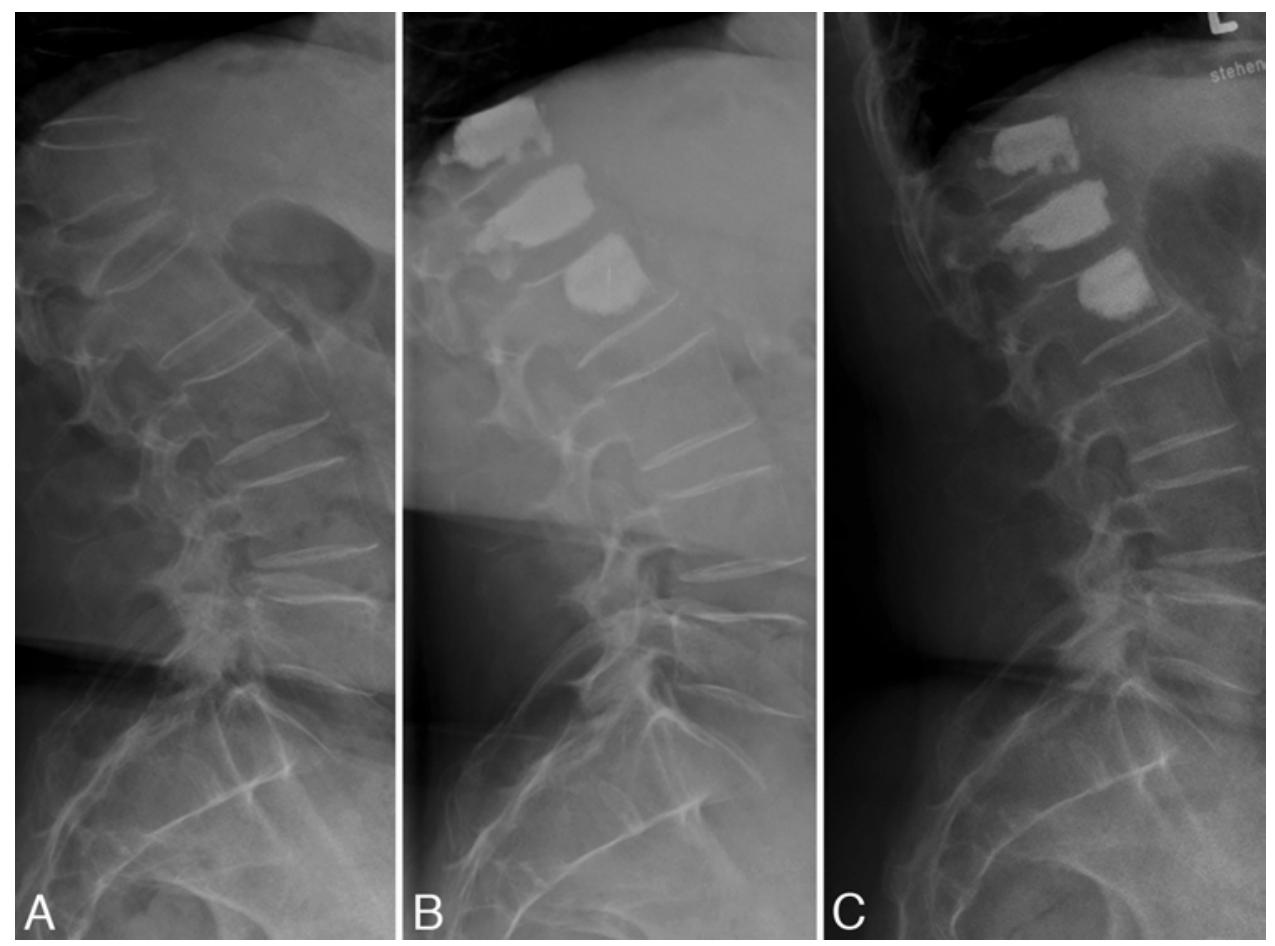

FIG. 2. Standing lateral radiographs obtained in a 65-year-old woman with a severe osteoporotic L-1 compression fracture. A: Preoperative image. B: Image obtained 6 weeks postoperatively showing good restoration of VKA and BKA after lordoplasty at L-1 and adjacent augmentation of T-12 and L-2. C: Image taken 3 years after the index operation. Persistent restoration of the initially restored VKA and BKA is shown, but unfortunately an adjacent compression fracture of T-11 has occurred.

loss of reduction below $1^{\circ}$. Orler et al ${ }^{25}$ reported a $15.2^{\circ}$ and $10^{\circ}$ correction of the VKAs and SKAs, respectively, at the 8-week follow-up. Similar results with a mean correction of $14^{\circ}$ of the VKA and $13^{\circ}$ of the SKA were reported in a case series by Jeon et al. ${ }^{14}$ In a retrospective study comparing lordoplasty and BKP, Kim et al..$^{15}$ found $11.4^{\circ}$ of VKA correction after lordoplasty and $7.0^{\circ}$ after BKP, which were not significantly different. Other studies show a variable degree of correction of kyphotic deformity between $1.7^{\circ}$ and $6.6^{\circ}$ for vertebroplasty, $4.8^{\circ}$ and $9.9^{\circ}$ for BKP, and $5.6^{\circ}$ and $7.3^{\circ}$ for VBS..$^{3,9,13,18,22,29}$ Deflation of the balloon results in a loss of $25 \%$ of vertebral body height. ${ }^{24}$ Voggenreiter et al. ${ }^{30}$ described a spontaneous mean reduction of VCF of $6.5^{\circ}$ due to placement of the patient prone only. The authors attributed this effect to dynamic fracture mobility in which BKP added $3.4^{\circ}$ to a kyphosis reduction. In a comparison with the last available standing radiograph, the authors also observed a significant mean loss of reduction of $3.1^{\circ}$. There is an ongoing controversy over whether restoration or at least improvement of sagittal alignment in compression fractures actually affects the clinical outcome. Kyphotic deformity after VCFs can potentially lead to compromise in pulmonary function, and, possibly also gastrointestinal function, causing more than pain and postural difficulties. It has been reported that 1 thoracic VCF can cause enough overall sagittal kyphosis to cause a $9 \%$ loss of forced vital capacity.

The restoration of normal overall spinal sagittal alignment in the elderly patient with VCFs and kyphotic deformity has therefore potentially theoretical benefits.
Although for kyphoplasty significant reduction of local kyphosis has been reported in the literature, to our knowledge there have been no reports on whether this leads to an improved overall sagittal alignment. We have observed that in our patients, even if lordoplasty reduced the fractured vertebra effectively, the overall sagittal alignment is not significantly altered by the procedure.

Thirty-five percent of our patients suffered a new fracture during a cumulative period of 9 years. This is a higher percentage than the reported rate for a new fracture in the literature, which varies between $17 \%$ and $27 \%$, based on different observation periods..$^{15,20-22}$ In a large cohort from the SWISSspine registry, a $21 \%$ rate for new spine fractures during 1 postoperative year after BKP was observed..$^{12}$ In a subcohort with monosegmental osteoporotic fractures from the SWISSspine registry, Spross et al. revealed a preoperative segmental kyphosis $>30^{\circ}$, or patients with comorbidities such as rheumatoid arthritis and a cardiovascular disease, to be at high risk of adjacent-segment fracture after BKP within 6 months after the index surgery. ${ }^{28}$ Thirteen percent of our cohort had a segmental kyphosis over $30^{\circ}$, and therefore had a particularly higher risk of adjacent-segment fracture according to the study by Spross et al. ${ }^{28}$ It is known that the cemented vertebra alters the modulus of stiffness. ${ }^{8,11,16}$ Consequently, some authors suggested the prophylactic adjacent vertebral body cementation of adjacent levels to have a protective effect. ${ }^{2}$ In all our osteoporotic patients and in 1 traumatic patient with osteopenic bone, an additional cement augmentation of the adjacent levels was performed to prevent 
a cutting through of the cannulas when applying the lordotic momentum. This may explain the relatively low rate of adjacent-segment fractures (12\%) as compared with other studies. ${ }^{3,6,20-22}$ Spross et al. observed a $10 \%$ rate of adjacent-vertebra fracture until 6 months postoperatively, whereas we found this same rate after an average of 33 months. ${ }^{28}$

Indications for a reoperation other than a new fracture were rare: 4 patients developed a painful segmental instability and required an instrumented fusion between 6 and 37 months postoperatively. The explanation for this type of complication is not obvious. An intervention-related damage to the stabilizing spine structures is rather improbable but not impossible.

The cement leakage rate assessed on immediate postoperative plain radiography was $10 \%$, which is rather low if compared with the literature reporting as much as $41 \%$ cement leakage in vertebral augmentation. ${ }^{5,6,13,15}$ The retrospective study by Kim et al. observed cement leakage in $50 \%$ of the 12 vertebrae with lordoplasty and in $27 \%$ of 26 vertebrae with BKP. The cement leakage rate after BKP in the SWISSspine registry was 22\%. ${ }^{12}$ An assessment using CT would have probably resulted in a higher cement leakage rate.

Our patients showed a significant reduction of pain and high patient satisfaction with the treatment $(98 \%$ of the patients would undergo the treatment again). These results are similar to those after vertebroplasty and BKP, but obviously these data must be interpreted carefully due to their retrospective nature. ${ }^{3,4,17}$

We did not perform a cost-effectiveness analysis in our study, but lordoplasty has relatively low material costs. When compared with a single-level BKP or VBS, the material cost for lordoplasty in Switzerland is less. There are, of course, large regional variations regarding the implant prices, and the balloons can be used for multiple levels if required (although not recommended by the producers). The duration for surgery in lordoplasty is slightly longer as compared with single-level vertebroplasty or BKP but remains reasonably short with an average of 55 minutes.

Some limitations of the study deserve to be mentioned. The present study is intrinsically limited due to its retrospective nature and the lack of a control group. Moreover, the impact of restoration of sagittal alignment on mid- and long-term clinical outcome remains unclear. Nevertheless, this also represents the largest case series with the longest follow-up duration describing this alternative augmentation technique.

\section{Conclusions}

Lordoplasty is an alternative, safe, minimally invasive method to reduce pain, restore vertebral height, and correct kyphotic deformity in cases of osteoporotic fractures in the midterm. The outcome of lordoplasty is comparable, but not favorable, to vertebroplasty and kyphoplasty. Whether the restoration of local kyphosis has a clinical benefit in the long term cannot be judged at this moment. This treatment approach might possess other advantages such as lower cost in comparison with other balloon-assisted, cement-augmentation procedures.

\section{References}

1. Barr JD, Barr MS, Lemley TJ, McCann RM: Percutaneous vertebroplasty for pain relief and spinal stabilization. Spine (Phila Pa 1976) 25:923-928, 2000

2. Chiang CK, Wang YH, Yang CY, Yang BD, Wang JL: Prophylactic vertebroplasty may reduce the risk of adjacent intact vertebra from fatigue injury: an ex vivo biomechanical study. Spine (Phila Pa 1976) 34:356-364, 2009

3. Diel P, Freiburghaus L, Röder C, Benneker LM, Popp A, Perler G, et al: Safety, effectiveness and predictors for early reoperation in therapeutic and prophylactic vertebroplasty: short-term results of a prospective case series of patients with osteoporotic vertebral fractures. Eur Spine J 21 (Suppl 6):S792-S799, 2012

4. Eck JC, Nachtigall D, Humphreys SC, Hodges SD: Comparison of vertebroplasty and balloon kyphoplasty for treatment of vertebral compression fractures: a meta-analysis of the literature. Spine J 8:488-497, 2008

5. Ha KY, Kim KW, Kim YH, Oh IS, Park SW: Revision surgery after vertebroplasty or kyphoplasty. Clin Orthop Surg 2:203-208, 2010

6. Hartmann F, Gercek E, Leiner L, Rommens PM: Kyphoplasty as an alternative treatment of traumatic thoracolumbar burst fractures Magerl type A3. Injury 43:409-415, 2012

7. Heini PF: The current treatment-a survey of osteoporotic fracture treatment. Osteoporotic spine fractures: the spine surgeon's perspective. Osteoporos Int 16 (Suppl 2):S85S92, 2005

8. Heini PF, Berlemann U, Kaufmann M, Lippuner K, Fankhauser C, van Landuyt P: Augmentation of mechanical properties in osteoporotic vertebral bones-a biomechanical investigation of vertebroplasty efficacy with different bone cements. Eur Spine J 10:164-171, 2001

9. Heini PF, Orler R: Kyphoplasty for treatment of osteoporotic vertebral fractures. Eur Spine J 13:184-192, 2004

10. Heini PF, Wälchli B, Berlemann U: Percutaneous transpedicular vertebroplasty with PMMA: operative technique and early results. A prospective study for the treatment of osteoporotic compression fractures. Eur Spine J 9:445-450, 2000

11. Higgins KB, Harten RD, Langrana NA, Reiter MF: Biomechanical effects of unipedicular vertebroplasty on intact vertebrae. Spine (Phila Pa 1976) 28:1540-1548, 2003

12. Hübschle L, Borgström F, Olafsson G, Röder C, Moulin P, Popp AW, et al: Real-life results of balloon kyphoplasty for vertebral compression fractures from the SWISSspine registry. Spine J 14:2063-2077, 2014

13. Hulme PA, Krebs J, Ferguson SJ, Berlemann U: Vertebroplasty and kyphoplasty: a systematic review of 69 clinical studies. Spine (Phila Pa 1976) 31:1983-2001, 2006

14. Jeon TS, Kim SB, Park WK: Lordoplasty: an alternative technique for the treatment of osteoporotic compression fracture. Clin Orthop Surg 3:161-166, 2011

15. Kim SB, Jeon TS, Lee WS, Roh JY, Kim JY, Park WK: Comparison of kyphoplasty and lordoplasty in the treatment of osteoporotic vertebral compression fracture. Asian Spine J 4:102-108, 2010

16. Kinzl M, Benneker LM, Boger A, Zysset PK, Pahr DH: The effect of standard and low-modulus cement augmentation on the stiffness, strength, and endplate pressure distribution in vertebroplasty. Eur Spine J 21:920-929, 2012

17. Klazen CA, Lohle PN, de Vries J, Jansen FH, Tielbeek $\mathrm{AV}$, Blonk MC, et al: Vertebroplasty versus conservative treatment in acute osteoporotic vertebral compression fractures (Vertos II): an open-label randomised trial. Lancet 376:1085-1092, 2010

18. Klezl Z, Majeed H, Bommireddy R, John J: Early results after vertebral body stenting for fractures of the anterior column of the thoracolumbar spine. Injury 42:1038-1042, 2011 
19. Kuklo TR, Polly DW, Owens BD, Zeidman SM, Chang AS, Klemme WR: Measurement of thoracic and lumbar fracture kyphosis: evaluation of intraobserver, interobserver, and technique variability. Spine (Phila Pa 1976) 26:61-66, 2001

20. Lin WC, Cheng TT, Lee YC, Wang TN, Cheng YF, Lui CC, et al: New vertebral osteoporotic compression fractures after percutaneous vertebroplasty: retrospective analysis of risk factors. J Vasc Interv Radiol 19:225-231, 2008

21. Lindsay R, Burge RT, Strauss DM: One year outcomes and costs following a vertebral fracture. Osteoporos Int 16:7885,2005

22. Lindsay R, Silverman SL, Cooper C, Hanley DA, Barton I, Broy SB, et al: Risk of new vertebral fracture in the year following a fracture. JAMA 285:320-323, 2001

23. Magerl F, Aebi M, Gertzbein SD, Harms J, Nazarian S: A comprehensive classification of thoracic and lumbar injuries. Eur Spine J 3:184-201, 1994

24. McKiernan F, Jensen R, Faciszewski T: The dynamic mobility of vertebral compression fractures. J Bone Miner Res 18:24-29, 2003

25. Orler R, Frauchiger LH, Lange U, Heini PF: Lordoplasty: report on early results with a new technique for the treatment of vertebral compression fractures to restore the lordosis. Eur Spine J 15:1769-1775, 2006

26. Pérez-Higueras A, Alvarez L, Rossi RE, Quiñones D, AlAssir I: Percutaneous vertebroplasty: long-term clinical and radiological outcome. Neuroradiology 44:950-954, 2002

27. Ploeg WT, Veldhuizen AG, The B, Sietsma MS: Percutaneous vertebroplasty as a treatment for osteoporotic vertebral compression fractures: a systematic review. Eur Spine J 15:1749-1758, 2006

28. Spross C, Aghayev E, Kocher R, Röder C, Forster T, Kuel- ling FA: Incidence and risk factors for early adjacent vertebral fractures after balloon kyphoplasty for osteoporotic fractures: analysis of the SWISSspine registry. Eur Spine J 23:1332-1338, 2014

29. Theodorou DJ, Theodorou SJ, Duncan TD, Garfin SR, Wong WH: Percutaneous balloon kyphoplasty for the correction of spinal deformity in painful vertebral body compression fractures. Clin Imaging 26:1-5, 2002

30. Voggenreiter G: Balloon kyphoplasty is effective in deformity correction of osteoporotic vertebral compression fractures. Spine (Phila Pa 1976) 30:2806-2812, 2005

\section{Disclosures}

The authors report no conflict of interest concerning the materials or methods used in this study or the findings specified in this paper.

\section{Author Contributions}

Conception and design: Hoppe, Bissig, Benneker. Acquisition of data: Budmiger, Bissig. Analysis and interpretation of data: all authors. Drafting the article: Hoppe, Aghayev. Critically revising the article: Hoppe, Bissig, Aghayev, Benneker. Approved the final version of the manuscript on behalf of all authors: Hoppe. Statistical analysis: Aghayev. Administrative/technical/material support: Hoppe, Budmiger, Bissig. Study supervision: Benneker.

\section{Correspondence}

Sven Hoppe, Department for Orthopedic Surgery, Spine Unit, Inselspital, University Hospital of Bern, Freiburgstrasse, Bern 3010, Switzerland. email: svenhoppe@gmail.com. 